\title{
ON RING STRUCTURES DETERMINED BY GROUPS
}

\author{
KLAUS E. ELDRIDGE
}

In this paper we show that part of the structure of an artinian ring is determined when it has a solvable, simple, nilpotent, supersolvable, torsion, or finitely generated quasi-regular group. For the case of a simple quasi-regular group, the rings are completely described. We also give an application of our results to finite dimensional group algebras.

Our proofs depend, in part, on properties of matrix algebras over division rings and the following two known lemmas.

Lemma 1. Let $R$ be an associative ring and let $n$ be a fixed natural number such that

(i) $R=M_{1} \oplus M_{2} \oplus \cdots \oplus M_{n}$ (ideal direct sum), then

(ii) ${ }^{\circ} R={ }^{\circ} M_{1} \otimes{ }^{\circ} M_{2} \otimes \cdots \otimes^{\circ} M_{n}$ (group direct product). Furthermore,

(iii) $\left|{ }^{\circ} R\right|=\prod_{1}^{n}\left|{ }^{\circ} M_{i}\right|$, and hence $\left.\right|^{\circ} R \mid<\infty$ if and only if $\left|{ }^{\circ} M_{i}\right|$ $<\infty$ for each $i$.

LeMma 2 (SATZ 4 of [8]). Every artinian ring is the direct sum of its torsion ideal and a torsion free ideal whose additive group is divisible.

Throughout the paper ${ }^{\circ} R$ denotes the quasi-regular group of the ring, where the operation is taken to be $a \circ b=a+b+a b$, while $J$ will be the Jacobson radical of $R$, and $|M|$ is the number of elements in the set $M$. If $R$ has a unity, then ${ }^{*} R$ will denote its group of units, in which case we also have ${ }^{\circ} R \simeq{ }^{*} R$.

1. Solvable or simple groups. We begin this section, in which we consider rings whose quasi-regular groups are solvable, nilpotent, or simple, with the following observation:

Proposition 1. For any ring $R$, the group ${ }^{\circ} R$ is solvable if and only if the groups ${ }^{\circ} R / J$ and ${ }^{\circ} J$ are solvable.

This follows directly from a known theorem for solvable groups and that the ring epimorphism $R \rightarrow R / J$ induces a group epimorphism ${ }^{\circ} R \rightarrow{ }^{\circ} R / J$.

Applying this observation to artinian rings gives us

Presented to the Society, August 30,1968, under the title Ring structures determined by groups. II ; received by the editors September 24, 1968 and, in revised form, May 26, 1969. 
THEOREM 1. For an artinian ring $R$, the group ${ }^{\circ} R$ is solvable if and only if the direct summands of $R / J$ are fields or isomorphic to the ring of $2 \times 2$ matrices over $G F(2)$.

Proof. The Wedderburn-Artin structure theorem says

$$
R / J=M_{1} \oplus M_{2} \oplus \cdots \oplus M_{n}
$$

for suitable matrix algebras $M_{i}$. By Proposition $1,{ }^{\circ} R / J$ is a solvable group. Hence the underlying division rings $\Delta_{i}$ must have solvable groups ${ }^{\circ} \Delta_{i} \simeq{ }^{*} \Delta_{i}$. But Hua shows in [5] that the only division rings with solvable multiplicative groups are fields. Furthermore, the ring of $2 \times 2$ matrices over $G F(2)$ is the only matrix ring with a solvable linear group (see Chapter 4 of [1]). Hence either $M_{i}$ is a field or the specified matrix ring, proving the "only if" part.

For the "if" part, observe that for any nilpotent ring $J$, the normal series

$$
{ }^{\circ} J>{ }^{\circ} J^{2}>\cdots>{ }^{\circ} J^{k}=(0),
$$

which is the same as the ideal chain

$$
J \supset J^{2} \supset \cdots \supset J^{k}=(0),
$$

has abelian factor groups. Thus ${ }^{\circ} \mathrm{J}$ is a solvable group. From the structure of $R / J$ we know, by Lemma 1 , that ${ }^{\circ} R / J$ is solvable. Applying Proposition 1 completes the proof.

Since the quasi-regular group of the ring of $2 \times 2$ matrices over $G F(2)$ is not nilpotent (it is isomorphic to the permutation group on 3 letters), we have the following corollary to Theorem 1.

CoROllaRY 1. For a semisimple artinian ring, ${ }^{\circ} R$ is nilpotent if and only if $R$ is a direct sum of finitely many fields.

When ${ }^{\circ} R$ is simple, the theorem yields another corollary which completely characterizes $R$.

COROLLARY 2. When the quasi-regular group of an artinian ring $R$ is simple, then $R$ is finite and has one of the following structures:

(a) $G F(2) \oplus G F(2) \oplus \cdots \oplus G F(2)$;

(b) $G F(3)$;

(c) $G F\left(2^{m}\right)$, $m$ such that $2^{m}-1$ is prime;

(d) $\left\{0, u, 2 u, \cdots,(p-1) u \mid u^{2}=0=p u\right\}$, where $p$ is any prime.

Proof. Clearly $R=J$ or $J=(0)$, since $J={ }^{\circ} J$ is a normal subgroup of ${ }^{\circ} R$. If $J=(0)$, the choice of fields is obvious. If $R=J$, then $J^{2}=(0)$, since $J^{2}={ }^{\circ} J^{2}$ is a normal subgroup of ${ }^{\circ} J$. Hence ${ }^{\circ} \mathrm{J}$ is elementary abelian and the result follows. 
2. Finitely generated groups. In this section we variously assume that the group ${ }^{\circ} R$ is finitely generated, super-solvable, torsion, or a combination of these, and pose 3 open problems.

We begin with a "negative" result.

Proposition 2. If $R$ is a torsion-free artinian ring, then ${ }^{\circ} R$ cannot be a finitely generated group.

Proof. Suppose ${ }^{\circ} R$ is a finitely generated group, then so is ${ }^{\circ} R / J$. Assume $R \neq J$. The Wedderburn-Artin structure theorem leads to an equation of type (i) of Lemma 1 for $R / J$ with each $M_{j}$ having a finitely generated group ${ }^{\circ} M_{j}$. Fix $j$ and let $\Delta$ be the underlying division ring. Using Dieudonné's determinant map [3], we induce an epimorphism of ${ }^{\circ} M_{j}$ onto the multiplicative group of the center $F$ of $\Delta$. Thus $* F$ is a finitely generated group, and hence $F$ must be a finite field. Therefore $R / J$ has finite characteristic, i.e. has torsion, which is impossible by Lemma 2 . However, if $R=J$ we have another contradiction, for Hopkins has shown in [4] that nilpotent artinian rings are torsion rings. Hence the proposition follows.

A different condition on ${ }^{\circ} R$ which implies $R$ is a torsion ring is given in

Proposition 3. If the quasi-regular group of an artinian ring is a torsion group, the ring is a torsion ring.

Proof. Denote the torsion-free component of $R$ in Lemma 2 by $R_{F}$. Suppose that $b \neq 0 \in J_{F}$, the radical of $R_{F}$. Since ${ }^{\circ} J_{F}$ is also a torsion group, there exists an integer $m$ such that $m b \in J_{F}^{2}$. Inductively we find an integer $k$ such that $k b \in J_{F}^{n}=(0)$, contradicting that $R_{F}$ was torsion-free. Hence $R_{F}$ is semisimple.

To see that $R_{F}=(0)$, let $\Delta$ be any division ring belonging to $R_{F}$ $\neq(0)$. Then ${ }^{\circ} \Delta$ is a torsion group and so is the center of $* \Delta$. If the characteristic of $\Delta$ is 0 , then its center contains a copy of the rational numbers whose multiplicative group is obviously not a torsion group. Hence $\Delta$ has finite characteristic-another contradiction. Thus $R_{F}=(0)$ and $R$ is a torsion ring.

REMARK. The condition of $R$ being artinian cannot be dropped in these two propositions as is readily seen by looking at the integers whose quasi-regular group has only 2 elements.

For the main theorem of this section we also need

LEMma 3. Let $R$ be an artinian ring with finite characteristic. If $1<|R / J|<\infty$, then $|J|<\infty$.

Proof. Since the characteristic of $R$ is finite, no element of the 
additive group of $R$ has infinite height. Hence, by Kaplansky's version of a theorem of Hopkins [7, Theorem 34, p. 52], $R$ is also a Noetherian ring. Therefore each one-sided ideal $K$ of $R$ is a finitely generated $R / A$-module, where $A$ is the annihilator of $K$ in $R$. Thus, if $J^{2}=(0), J$ is a finitely generated $R / J$-module and hence finite. Using induction, we assume that $R /\left(J^{r-1}\right)=B$ is finite, where $r>2$ is the index of nilpotence of $J$. Then $J$ is a finitely generated $B$-module, and hence finite as a $R$-module, proving the lemma.

THEOREM 2. Let $R$ be an artinian ring such that ${ }^{\circ} R$ is a finitely generated group and ${ }^{\circ} R / J$ is a torsion group. Then $R$ must be a finite ring.

Proof. By Proposition 2, $R$ is a torsion ring. Let $\left\{u_{1}, u_{2}, \cdots, u_{t}\right\}$ be the generators of ${ }^{\circ} R$ and let $\left\{m_{1}, m_{2}, \cdots, m_{t}\right\}$ be the corresponding additive orders of the $u_{i}$. Since every element of $J$ is a polynomial in the $u_{i}$ 's, it must have additive order dividing $\prod_{1}^{t} m_{i}=m$. Hence $J$ has characteristic $k \mid m$. If $R / J=(0)$, we are done. For the total degree of these polynomials in the $u_{i}$ 's is bounded by the index of nilpotence, and the coefficients are integers modulo $k$.

If $R / J \neq 0$, then $R / J$ has finite characteristic $q$, since $R / J$ is a torsion ring by Proposition 3 and also has a unity. Hence $R$ has characteristic $q k$.

Since ${ }^{\circ} R / J$ is a torsion group, every division ring $\Delta$ belonging to $R / J$ is a field by the commutativity theorem for division rings [6, Theorem 2, p. 183]. With the aid of the determinant map, we now see that each field $\Delta$ has a finitely generated group ${ }^{*} \Delta \simeq^{\circ} \Delta$, by following the epimorphs

$$
{ }^{\circ} R \rightarrow{ }^{\circ} R / J \rightarrow{ }^{\circ} \Delta .
$$

But only finite fields have finitely generated multiplicative groups. So $R / J$ is finite and Lemma 3 completes the proof.

Recalling that a super-solvable group is a finitely generated solvable group, we have the following corollary to the theorem after applying Theorem 1 to $R / J$.

COROLLARY 1. If $R$ is an artinian ring with a super-solvable quasiregular group, then $R$ is finite.

We also have

Corollary 2. Let $R$ be an artinian ring. Suppose that ${ }^{\circ} R$ is a finitely generated nilpotent group. Then ${ }^{\circ} R \simeq^{\circ} J \otimes A$, where $A$ is a subgroup of the center of ${ }^{\circ} R$. Conversely, if ${ }^{\circ} R \simeq^{\circ} J \otimes A$, then ${ }^{\circ} R$ is nilpotent. 
Proof. If ${ }^{\circ} R$ is finitely generated and nilpotent, $R / J$ is finite by Corollary 1 to Theorem 1 . Hence $R$ is finite by Theorem 2 . Write $R=R_{1} \oplus R_{2} \oplus \cdots \oplus R_{n}$, where $\left|R_{i}\right|=p_{i}^{c_{i}}$ with the $p_{i}$ ranging over the distinct prime divisions of $|R|$. Since ${ }^{\circ} R$ is now a finite nilpotent group, it is the direct product of its Sylow subgroups. Note that $R_{i} / J_{i}$ is a direct sum of finite fields of characteristic $p_{i}$ by Corollary 1 to Theorem 1. So the $p_{i}$-Sylow subgroup of ${ }^{\circ} R_{i}$ is precisely ${ }^{\circ} J_{i}$, while the $p_{i}$-complement $A_{i}$ of ${ }^{\circ} R_{i}$ is necessarily abelian and thus in the center of ${ }^{\circ} R_{i}$. Hence ${ }^{\circ} R_{i} \simeq^{\circ} J_{i} \otimes A_{i}$. From

$$
{ }^{\circ} R={ }^{\circ} R_{1} \otimes \cdots \otimes{ }^{\circ} R_{n} \text { and } J=J_{1} \oplus \cdots \oplus J_{n}
$$

it follows by Lemma 1 that ${ }^{\circ} R \simeq^{\circ} J \otimes A$, where $A \simeq A_{1} \otimes \cdots \otimes A_{n}$ is in the center of ${ }^{\circ} R$.

Conversely, suppose that ${ }^{\circ} R \simeq^{\circ} J \otimes A$, where $A$ is in the center of ${ }^{\circ} R$. Denote the lower central series of ${ }^{\circ} R$ by $\gamma_{n}$ so that $\gamma_{1}={ }^{\circ} R$ and $\gamma_{n+1}=\left[\gamma_{n},{ }^{\circ} R\right]$ for $n \geqq 1$. Since ${ }^{\circ} R / J$ is abelian, $\gamma_{2}=\left[{ }^{\circ} R,{ }^{\circ} R\right] \subset^{\circ} J$. Now every $u \in{ }^{\circ} R$ has the form $u=b \circ a$ with $b \in J$ and $a \in A$. Thus for any $v \in J$ and $u \in^{\circ} R$ we have

$$
[v, u]=v^{\prime} \circ u^{\prime} \circ v \circ u=v^{\prime} \circ b^{\prime} \circ v \circ b,
$$

where primes denote inverses in ${ }^{\circ} R$. But the element on the right side of this equation belongs to ${ }^{\circ} J^{2}$. Hence we have $\gamma_{3} C^{\circ} J^{2}$. Inductively, it follows that

$$
\gamma_{n} \subset{ }^{\circ} J^{n-1} \text { for all } n>1 .
$$

Since ${ }^{\circ} J$ is nilpotent, we have $\gamma_{k}=(0)$ for some integer $k \geqq 2$. Hence ${ }^{\circ} R$ is nilpotent, completing the proof of the corollary.

REMARK. In connection with Theorem 2 and its corollaries we have three open questions, the second being a special case of the first:

1. If we drop the condition " $R / J$ is a torsion group" in Theorem 2 , do we still get $|R|<\infty$ ?

2. Let $\Delta$ be a division ring with a finitely generated group ${ }^{\circ} \Delta \simeq^{*} \Delta$. Is $\Delta$ necessarily a finite field?

3. If, in Corollary 2, we only assume that ${ }^{\circ} R$ is nilpotent, do we still obtain ${ }^{\circ} R \simeq^{\circ} J \otimes A$, where $A$ is in the center of ${ }^{\circ} R$ ?

3. Application. We now apply the results of the provious sections to group algebras extending a theorem of Bateman and Coleman [2] as well as providing a correct proof for their theorem.

THEOREM 3. Let $G$ be a finite group and let $F$ be a field. Let $\Gamma=\Gamma(G, F)$ be the group algebra of $G$ over $F$.

(1) If $\Gamma$ is semisimple, then ${ }^{\circ} \Gamma$ is nilpotent if and only if $G$ is abelian.

(2) If the characteristic $p \neq 0$ of $F$ divides the order of $G$, then ${ }^{\circ} \Gamma$ is 
nilpotent if and only if $G$ is nilpotent and every $q$-Sylow subgroup of $G$ is abelian for $q \neq p$.

The proof of part (1) follows directly from Corollary 1 of Theorem 1 , since $\Gamma$ is an artinian ring.

The proof of part (2). Suppose that ${ }^{\circ} \Gamma$ is nilpotent. Since $G$ is finite, we have, by Corollary 2 of Theorem 2 , that ${ }^{\circ} \Gamma \simeq^{\circ} J \otimes A$. Since $G$ is isomorphic to a subgroup of ${ }^{\circ} \Gamma$, it is nilpotent and every $q$-Sylow subgroup of $G$ is in the center of ${ }^{\circ} \Gamma$, and hence in the center of $G$ for $q \neq p$.

Conversely, suppose that $G$ is nilpotent. Then $G=P \otimes A$, where $P$ is the $p$-Sylow subgroup of $G$ and $A$ is the abelian $p$-complement of $G$. Since $\Gamma(A, F)$ is semisimple, we have by part (1)

$$
\Gamma(A, F)=F_{1} \oplus F_{2} \oplus \cdots \oplus F_{n},
$$

where each $F_{i}$ is a finite extension field of $F$. Now $\Gamma(G, F) \simeq \Gamma(A, F)$ $\otimes_{F} \Gamma(P, F)$, and since tensor products distribute over direct sums, we need only consider $F_{i} \otimes_{F} \Gamma(P, F)$ for a fixed $i$. Set $\Gamma_{i}=\Gamma\left(P, F_{i}\right)$ $\simeq F_{i} \otimes_{F} \Gamma(P, F)$. Note that each $u \in^{\circ} \Gamma_{i}$ has the form $u=\alpha+b$, where $\alpha \in F_{i}$ and $b \in^{\circ} J_{i}$. Hence ${ }^{\circ} \Gamma_{i}$ is nilpotent by Corollary 2 of Theorem 2, since ${ }^{\circ} F_{i}$ is in the center of ${ }^{\circ} \Gamma_{i}$ and ${ }^{\circ} \Gamma_{i} \simeq^{\circ} J_{i} \otimes{ }^{\circ} F_{i}$. The rest follows from the fact that

$$
{ }^{\circ} \Gamma \simeq{ }^{\circ} \Gamma_{1} \otimes{ }^{\circ} \Gamma_{2} \otimes \cdots \otimes{ }^{\circ} \Gamma_{n},
$$

completing the proof of the theorem.

I want to thank the referee and Professor Kaplansky for their helpful suggestions and corrections which greatly improved this paper.

\section{REFERENCES}

1. Emil Artin, Geometric algebra, Wiley, New York, 1957.

2. J. M. Bateman and D. B. Coleman, Group algebras with nilpotent groups, Proc. Amer. Math. Soc. 19 (1968), 448-449.

3. Jean Dieudonné, Les dêterminants sur un corps noncommutatif, Bull. Soc. Math. France 71 (1943), 27-45.

4. Charles Hopkins, Nilrings with minimal condition, Duke Math. J. 4 (1938), 664-667.

5. Loo-Keng Hua, On the multiplicative group of a field, Acad. Sinica Science Record 3 (1950), 1-2.

6. Nathan Jacobson, Structure of rings, Amer. Math. Soc. Colloq. Publ., vol. 37, 2nd rev. ed., Amer. Math. Soc., Providence, R. I., 1964.

7. Irving Kaplansky, Notes on ring theory, rev. ed., Math Lecture Notes, University of Chicago, Chicago, Ill., 1965.

8. F. Szasz, Über Artinsche Ringe, Bull. Acad. Polon. Sci. Sér. Sci. Math. Astronom. Phys. 21 (1963), 351-354. 